\title{
ADECUACIÓN DE ENERGÍA Y NUTRIENTES E ÍNDICE DE ALIMENTACIÓN SALUDABLE EN MUJERES CLIMATÉRICAS
}

\section{ENERGY AND NUTRIENTS ADJUSTMENT AND HEALTHY EATING INDEX IN CLIMATERIC WOMEN}

\author{
Eliana Durán F. (1), Delia Soto A. (2), Ana María Labraña T. (1) Katia Sáez C. (3) \\ (1) Departamento de Bromatología, Nutrición y Dietética, Facultad de Farmacia, \\ Universidad de Concepción, Concepción, Chile. \\ (2) Facultad de Química y Farmacia, Universidad de Chile. Santiago, Chile. \\ (3) Departamento de Estadística, Facultad de Ciencias Físicas y Matemáticas, \\ Universidad de Concepción, Concepción, Chile.
}

\begin{abstract}
Diet quality was described analyzing the energy and nutrients adjustment and the Healthy Eating Index (HEI) in 23 climacteric women from a universe of 268. Their ages ranged from 47 to 61 years old and they worked in the administration division of the University of Concepcion. Dietetic evaluation was carried out using the survey technique, registering the daily food consumption during 7 consecutive days, separating the registers according to two seasonal periods: Spring-Summer and Autumn-Winter. It was observed a normal calorie adjustment in 12 of 23 women, while carbohydrates, monounsaturated fatty acids, cholesterol, calcium and fiber showed a significant lower adjustment. In contrast proteins, vitamin $C$ and iron showed a higher adjustment. The HEI average score at both seasons was 61.8 \pm 12.5 ( $p>0.05$ ) classifying the feeding as "change necessity" and the feeding goals were achieved in less than $65 \%$. The lower score corresponded to carbohydrates, dairy products and fruits. It is possible to conclude that women feeding require a food intervention in order to improve the nutrients adjustment and the Healthy Eating Index.
\end{abstract}

Key words: climacteric women, food intake survey, nutrients, Healthy Eating Index.

Este trabajo fue recibido el 26 de Octubre de 2007 y aceptado para ser publicado el 25 de Agosto de 2008.

\section{INTRODUCCIÓN}

El climaterio es una etapa en la vida de la mujer que la OMS sitúa entre los 45 y 64 años (1). El aumento de las expectativas de vida observado en las mujeres de países desarrollados también se presenta en Chile (2-4). Esto condiciona que sea de la mayor relevancia sanitaria conocer las diferentes características de la calidad de vida en la mujer climatérica chilena, las que el año 2010 constituirán el 12,2 \% de la población (5). La menopausia, cese ininterrumpido de la menstruación de más de 12 meses, constituye la manifestación fundamental del climaterio. Los cambios endocrinológicos que ocurren durante el climaterio y la menopausia, determinan cambios en el metabolismo, balance energético y en la composición corporal (6).

Las necesidades nutricionales en el climaterio y la menopausia tienen características de importancia debido al aumento de los factores de riesgo para enfermedades tales como las cardiovasculares, el inicio de osteoporosis, osteomusculares, cáncer y enfermedades seniles. Cuando no existen complicaciones metabólicas asociadas, la dieta en la menopausia se suscribe como una alimentación suficiente y equilibrada en función de la edad, talla, composición personal, clima y actividad física (7). Es fundamental en esta etapa de riesgo metabólico, disminuir progresivamente el aporte de calorías diarias, aumentar el aporte de calcio y limitar el aporte de grasas saturadas debido a que está demostrado que empeoran la resistencia a la insulina por un fenómeno de regulación descendente del receptor de insulina y los efectos conocidos sobre el receptor $\operatorname{LDL}(8,6)$.

A través de una correcta alimentación la mujer 
climatérica puede prevenir patologías logrando un envejecimiento saludable y cumpliendo de esta forma, con los objetivos sanitarios propuestos por el Ministerio de Salud de Chile para la década. La educación alimentaria y nutricional considerando los factores socioculturales, debe enfocarse a este grupo de edad que en Chile presenta un aumento debido a las mayores expectativas de vida de las mujeres esperado para el quinquenio 20052010, en 81 años (9).

Al respecto, desde el año 2001 se cuenta en Chile con las Guías Alimentarias para la Mujer las que están orientadas a que alcance y mantenga un adecuado estado nutricional en todas las etapas de su vida haciéndose necesaria una evaluación del impacto de estas Guías $(10,11)$.

El climaterio puede suponer una etapa de consolidación de los hábitos alimentarios que practicado de forma correcta ayuden a prevenir y a moderar algunos problemas como lo ya descritos (2-4). Diseñar, realizar o evaluar programas de salud que pretendan lograr mejorías en la calidad de vida de las mujeres, requiere del desarrollo de instrumentos adecuados para esos objetivos $(12,13)$. En este sentido indicadores dietéticos como el grado de suficiencia de la dieta (adecuación de calorías y nutrientes críticos) y el índice de alimentación saludable (IAS) en distinta época del año, deberían ser descritos para este grupo de edad ya que pueden constituir un aporte valioso al diagnóstico alimentario nutricional, permitiendo la identificación de factores alimentarios protectores de la salud o de riesgo de enfermar (14).

El presente estudio tuvo como objetivo describir la calidad de la dieta desde el punto de vista cuantitativo y cualitativo analizando el grado de suficiencia de la dieta a través de la adecuación de energía y nutrientes, y el índice de alimentación saludable respectivamente, en un grupo de mujeres en etapa de climaterio.

\section{SUJETOS Y METODO}

Este estudio de casos con un diseño prospectivo se realizó en los años 2002-2004 en un grupo de 23 mujeres de edad entre 47 a 61 años, seleccionadas al azar, de un universo de 268 , pertenecientes al estamento administrativo de la Universidad de Concepción.

Los requisitos para participar en el estudio fueron, escolaridad igual o superior a 14 años, disponibilidad para registrar su dieta por 7 días 2 veces en un año y ausencia de patología diagnosticada por médico, determinante de su alimentación. Posterior a una invitación escrita realizada a 80 mujeres que cumplieron los requisitos de edad, se procedió a realizar una entrevista personal a 25 de ellas quienes cumplieron con los criterios de inclusión, para darles a conocer el objetivo del estudio y la forma de participar. Las participantes firmaron el consentimiento libre e informado exigido por el Comité de Etica de la Facultad de Farmacia de la Universidad de Concepción.

Para conocer las características antropométricas del grupo, se realizaron las mediciones de talla y peso con cartabón (escala de 1 a $200 \mathrm{~cm}$ y precisión igual a 0,1 $\mathrm{cm}$ ) y una balanza marca Seca (modelo 220, con escala de 5 a $150 \mathrm{Kg}$ y precisión de $0,1 \mathrm{Kg}$ ) respectivamente, con estos valores se obtuvo el índice de masa corporal (IMC Kg/m2). La circunferencia de cintura se midió con huincha metálica inextensible graduada en centímetros. Los pliegues cutáneos se midieron con calibrador marca Lange; el porcentaje de grasa corporal obtenido por sumatoria de 4 pliegues, se clasificó según Durnin and Womersley (15).

Para calcular las necesidades energéticas individuales en función de la edad, se utilizó la recomendación FAO/OMS/UNU-1985, que considera peso esperado para la talla real (8). Para la adecuación energética se utilizó la referencia del National Research Council, 1989 que establece un rango adecuado entre 80 y $120 \%$ de lo recomendado (16). Los resultados se presentarán según aportes menores o mayores estadísticamente significativos respecto de las recomendaciones.

Para la evaluación dietética, se utilizó la técnica de encuesta de registro diario de consumo de alimentos por 7 días consecutivos, separando los registros en dos períodos estacionales del año: Primavera-Verano (PV) y Otoño-Invierno (OI). Se constituyeron 14 días de registro dietético en un tiempo aproximado de 1 año calendario por cada persona, totalizando 322 minutas. Se entregó el formulario de registro, instructivo escrito y oral sobre la forma para llenar el formulario y sobre el peso y medida casera de alimentos, preparaciones y bebidas.

Se facilitó a cada participante una balanza de precisión portátil marca SOEHNLE con capacidad de 0 a 2000 gramos y 1 gramo de precisión. Se dispuso de asistencia personalizada por Nutricionista para el adecuado registro, durante la semana objetivo del estudio, en ambos períodos estacionales.

Una vez recolectados los formularios se calcularon y analizaron: el aporte nutricional y la estructura alimentaria diaria con software Evaluación y Adecuación de la dieta de la Universidad de Concepción (17). Se calcularon los indicadores dietarios: porcentaje de adecuación calórica y de nutrientes críticos e índice de alimentación saludable, IAS, según el modelo sugerido (12). Este último incluye 10 variables con un máximo de 10 puntos cada una que sumadas pueden dar un máximo de 100 puntos: cinco variables están referidas a las porciones 
consumidas de los principales grupos de alimentos de la pirámide alimentaria (cereales, verduras, verduras, frutas, lácteos, carnes)(11), cuatro a las metas alimentarias (porcentaje de las calorías totales proveniente de los lípidos, grasas saturadas, azúcares, y gramos máximos de sodio en el que se consideró el sodio intrínseco y extrínseco equivalente a 2,4 gramos de sal) (12), y una variable se relaciona con la variedad en el consumo de los alimentos, en grupos de población.

Las porciones esperadas se presentan en la tabla 5. Se utilizó el siguiente rango de clasificación de la calidad de la dieta: $>80$ puntos, "Saludable"; $51-79$ puntos "Necesita cambios"; $<50$ puntos "Poco saludable" y de "prioridad de intervención".

Las adecuaciones de nutrientes se analizaron según recomendaciones internacionales $(16,18,19)$. Las metas alimentarias por porciones, se analizaron según las Guías Alimentarias para la Mujer recomendadas para 1800 Calorías diarias (11) debido a que las necesidades promedio de energía resultaron equivalentes a una dieta de esta característica (8).

Se creó una base de datos en Excel la cual fue analizada mediante ANOVA con mediciones repetidas. Para ello se empleó el software estadístico SAS, versión 9.1 (SAS Online Doc. versión 9.1. SAS Institute Inc. Cary, NC, USA. 2003). Se consideraron significativos valores de $p<0,05$. Se utilizó el test $t$ para determinar diferencias entre las recomendaciones de energía y nutrientes y los aportes promedio. Para el cálculo del IAS se determinó por comparación de medias y el coeficiente de correlación de Pearson $(\mathrm{p}<0,05)$, el día de la semana representativo de la alimentación por estacionalidad, resultando en el día Miércoles para IO (23 minutas) y Jueves para PV (23 minutas).

\section{RESULTADOS}

Se encontró el IMC en un amplio rango: de las 23 mujeres, 4 presentaron valores $\geq 30 \mathrm{Kg} / \mathrm{m} 2,10$ presentaron valores entre 25 y 29,9 y 9 presentaron valores entre 18,5 y $24,9 \mathrm{Kg} / \mathrm{m} 2$. Valores de grasa corporal $\geq$ $36 \%$, presentaron 14 mujeres. La talla media fue de $155,9 \mathrm{~cm} . \pm 4,1$ y el peso esperado para esa talla fue de $52,7 \mathrm{Kg}$. La circunferencia media de cintura fue de $82,5 \mathrm{~cm} . \pm 10,6$.

La adecuación calórica y nutritiva de la dieta global se presenta en la tabla 1 observándose una adecuación

\section{TABLA 1}

Adecuación calórica y de nutrientes críticos de la dieta global de las embarazadas.

\begin{tabular}{|c|c|c|c|c|c|}
\hline & $\begin{array}{c}\text { Recomendación } \\
\text { (1) }\end{array}$ & Promedio & $\begin{array}{c}\text { Aportes } \\
\text { Menor } \\
\text { n }\end{array}$ & $\begin{array}{c}\text { Adecuado } \\
\mathrm{n}\end{array}$ & $\begin{array}{c}\text { Mayor } \\
\mathrm{n}\end{array}$ \\
\hline Calorías & 1767 & 1720 & 6 & 12 & 4 \\
\hline Proteínas (g) & 57.6 & 67.7 & 2 & 12 & 11 \\
\hline Hidratos de carbono $(\mathrm{g})$ & 280 & 238.6 & 15 & 6 & 2 \\
\hline Lípidos (g) & 49 & 55 & 4 & 13 & 6 \\
\hline Saturados $(g)(2)$ & 13.7 & 15.9 & 3 & 16 & 4 \\
\hline Monosaturados (g) & 29.5 & 15.4 & 21 & 2 & 0 \\
\hline Polisaturados (g) & 15.7 & 12.2 & 10 & 13 & 0 \\
\hline Colesterol (mg) & 300 & 151.6 & 19 & 4 & 0 \\
\hline Vit C (mg) & 75 & 115.2 & 1 & 13 & 9 \\
\hline Hierro (mg) & 10 & 15.5 & 3 & 2 & 18 \\
\hline Calcio (mg) & 1500 & 692 & 22 & 1 & 0 \\
\hline Fibra (g) & 26 & 5.6 & 23 & 0 & 0 \\
\hline
\end{tabular}


normal de calorías para 12 de las 23 mujeres con adecuaciones significativamente menores en cuanto a hidratos de carbono, ácidos grasos monosaturados, colesterol, calcio y fibra dietética, para una proporción mayoritaria de las mujeres, y significativamente mayores en proteínas, vitamina $\mathrm{C}$ y hierro. El hierro de origen animal correspondió a 23,5\%.

El puntaje promedio del IAS en ambas estaciones fue de 61,8 \pm 12,5 clasificando la alimentación con "necesidad de cambio" y cumplimiento de las metas alimentarias inferior a $65 \%$. En la tabla 2 se presenta el puntaje promedio de cada uno de los componentes del IAS en cada estación del año con diferencias significati- vas de mayor consumo de frutas, de lácteos y de variedad de alimentos en otoño-invierno. Las metas alimentarias mejor logradas fueron para las carnes, grasas saturadas y sal (expresada en sodio) con puntajes superiores a 7 en la escala de 1 a 10. En tanto los puntajes más bajos correspondieron a azúcar con ingestas superiores al 10 $\%$ de las calorías totales diarias y a lácteos con ingestas promedio inferiores a 3 porciones diarias.

La distribución de mujeres según clasificación del IAS de acuerdo al puntaje total se presenta en la tabla 3 y se observa que una pequeña proporción de ellas tiene alimentación saludable, la mayoría necesita cambios y una proporción menor tiene alimentación

\section{TABLA 2}

\section{Indice de Alimentación Saludable según estaciones: Primavera-Verano (PV) y Otoño-Invierno (OI).}

\begin{tabular}{lccc}
\hline Componentes IAS* & PV promedio \pm DE & OI promedio \pm DE & p \\
\hline Cereales & $6,67 \pm 3,0$ & $7,8 \pm 2,3$ & $\mathrm{~ns}$ \\
Verduras & $6,09 \pm 3,3$ & $5,67 \pm 3,3$ & $\mathrm{~ns}$ \\
Frutas & $4,92 \pm 3,6$ & $6,06 \pm 2,9$ & $<0,05$ \\
Lácteos & $3,46 \pm 3,6$ & $4,91 \pm 2,8$ & $<0,05$ \\
Carnes & $8,54 \pm 3,1$ & $8,37 \pm 3,2$ & $\mathrm{~ns}$ \\
Grasas & $6,65 \pm 4,6$ & $6,87 \pm 4,7$ & $\mathrm{~ns}$ \\
Grasas saturadas & $7,70 \pm 4,1$ & $7.35 \pm 4,5$ & $\mathrm{~ns}$ \\
Azúcar & $0,87 \pm 2,9$ & $1,30 \pm 3,4$ & $\mathrm{~ns}$ \\
Sodio & $7,46 \pm 1,7$ & $7,46 \pm 1,6$ & $\mathrm{~ns}$ \\
Variedad & $5,94 \pm 1,6$ & $6,70 \pm 1,5$ & $<0,05$ \\
Total & $59,9 \pm 12,2$ & $63,8 \pm 13,6$ & $\mathrm{~ns}$ \\
\hline *IAS : Indice de Alimentación Saludable. & & & \\
\hline
\end{tabular}

\section{TABLA 3}

\section{Clasificación del Indice de Alimentación Saludable (IAS) por estacionalidad}

\section{CATEGORÍAS DE IAS}

Primavera - Verano

Otoño - Invierno

Saludable

Necesita cambios

Poco saludable 
poco saludable.

La tabla 4 muestra las porciones de alimentos esperadas con relación al promedio de las observadas según estacionalidad apreciándose diferencias significativas en las porciones observadas en primavera-verano con excepción de las grasas que están dentro de lo esperado. En cambio en otoño-invierno las porciones de cereales, fruta, carnes, grasas totales, grasas saturadas no presentan diferencias estadísticamente significativas, respecto de las porciones esperadas.

\section{DISCUSIÓN}

En este estudio de casos, aunque la muestra fue de sólo 23 mujeres, se refleja el estado nutricional de lo que en la actualidad se presenta en la población femenina mayor de 40 años en el país. La prevalencia de sobrepeso encontrada resultó similar a la reportada por otros estudios (20, 21-23). Sin embargo esta situación no presenta relación con el aporte calórico promedio de la dieta estudiada, el que aparece insuficiente (6 casos) o dentro de lo recomendado (12 casos). Lo anterior pudiera deberse a un bajo gasto energético debido al síndrome sarcopénico propio de esta edad y por la disminución progresiva del metabolismo basal que ocurre a medida que se avanza en la edad (6); que las recomendaciones pudieran estar sobreestimadas; que la ganancia de peso medida por IMC es una condición de periodos de tiempo prolongado y por lo tanto representan procesos crónicos, en este caso de sobrepeso y obesidad; el alto nivel de escolaridad de las encuestadas que les permite mayor acceso a la información sobre el contenido calórico de los alimentos y de esta manera preferir aquellos con menos calorías.

Se sabe que las necesidades energéticas están orientadas a conseguir el peso adecuado expresado en un índice de masa corporal entre 18,5 y $25 \mathrm{Kg} / \mathrm{m} 2$, rango asociado a menor morbilidad y a mayor longevidad (11). En este estudio el peso adecuado para la edad, determinado por la talla según lo establecido por FAO/OMS/UNU (8) resultó en $52.7 \mathrm{Kg}$, valor que no estuvo presente en ninguno de los casos estudiados. Este aspecto debería ser considerado en el momento de la consulta nutricional y en los programas sobre estilos de vida saludable, como una meta a lograr.

En cuanto a los aportes de la dieta, los macro nutrientes sugieren una preferencia por los alimentos proteicos los que alcanzaron una participación relativa en las calorías de entre 15 y $16 \%$ de acuerdo a datos ya publicados (14). Al respecto estudios sugieren que dicha preferencia pudiera ser perjudicial al generar aumento en la excreción renal de calcio, favoreciendo la osteoporosis factor que particularmente importante en la mujer climatérica que presenta progresiva pérdida de masa ósea (20).

\section{TABLA 4}

\section{Porciones de alimentos esperadas y promedio de las observadas según estacionalidad}

\begin{tabular}{|c|c|c|c|c|c|}
\hline $\begin{array}{c}\text { Observadas } \\
\text { Componentes IAS }\end{array}$ & esperadas & PV & $\mathbf{p}$ & OI & $\mathbf{p}$ \\
\hline Cereales & 5 & 3,6 & $*$ & 4,5 & ns \\
\hline Verduras & 4 & 3 & $*$ & 2,8 & $*$ \\
\hline Frutas & 3 & 1,9 & $*$ & 2,3 & ns \\
\hline Lácteos & 4 & 1,4 & $*$ & 2,1 & $*$ \\
\hline Carnes & 2 & 2,7 & $*$ & 2,5 & ns \\
\hline Grasas $^{1}$ & $15-30 \%$ & 28,6 & ns & 27,9 & ns \\
\hline Grasas saturadas $^{1}$ & $<10 \%$ & 7,6 & $*$ & 8,9 & $\mathrm{~ns}$ \\
\hline Azúcar ${ }^{1}$ & $<10 \%$ & 23,4 & $*$ & 21,8 & $*$ \\
\hline Sodio $^{2}$ & $<1,65 \mathrm{~g}$ & 2,2 & $*$ & 2,3 & $*$ \\
\hline Variedad & 10 & 5,9 & $*$ & 6,7 & $*$ \\
\hline
\end{tabular}


El consumo de hidratos de carbonos totales es bajo lo que explicaría los resultados del aporte calórico obtenido, ya que 15 de las 23 mujeres presentaron ingestas de hidratos de carbonos significativamente menores a las recomendadas. Situación contraria se observó en el consumo de azúcares, hidratos de carbono simples, con adecuaciones muy superiores al $10 \%$ de las calorías totales (valor límite máximo en el IAS), en ambas estaciones del año. El alto consumo de azúcares detectado y considerado como nutriente crítico por el Ministerio de Salud, hace necesaria su inclusión como factor de riesgo de enfermar en los futuros programas de educación alimentaria y en la consejeria nutricional (24). Este aspecto explicaría el estado nutricional sobre lo normal que presentó la mayoría de las mujeres estudiadas y el respectivo porcentaje de grasa corporal.

Las grasas saturadas y totales, aparecen mayoritariamente adecuadas, con ingestas inferiores al $10 \%$ de las calorías totales, coincidiendo con otro estudio nacional (12), por lo que se deduce que estas mujeres tienen conciencia del riesgo de enfermar por consumo excesivo de grasas debido probablemente a poseer mayor acceso a información nutricional y a su alto nivel educacional. Investigadores señalan que la grasa dietarea es determinante primaria de la obesidad, sin embargo su participación relativa en el valor calórico total de la dieta se ha reducido en los últimos años (20).

Aunque las porciones de verduras y frutas fueron inferiores a las recomendadas, los aportes de vitamina C fueron adecuados con promedio de $115.2 \mathrm{mg}$ diarios, presentándose una sola mujer con una ingesta inferior a $75 \mathrm{mg}$ al día, constituyendo un factor protector de la salud. Respecto de las frutas llama la atención su mayor consumo en Otoño-Invierno, y el análisis de las minutas indica un mayor consumo de ensaladas en Primavera -Verano como reemplazo de la fruta lo que explicaría la situación descrita.

Con relación al hierro los resultados muestran que la media es adecuada en $155 \%$ lo que se explica por el consumo de alimentos proteicos especialmente las 2,5 porciones carnes diarias, aunque el hierro hemínico corresponde sólo al 23,5\% el alto consumo de vitamina $\mathrm{C}$ garantiza una mejor absorción del hierro total. Es importante que este grupo de edad mantenga las porciones de consumo de carnes sugeridas en la Guías Alimentarias para asegurar la ingesta de hierro hemínico y alcanzar la recomendación de $10 \mathrm{mg} /$ día $(19,16)$.

La adecuación de calcio resultó significativamente menor a las recomendaciones, explicada por el consumo inferior a las 3 porciones de lácteos al día. El calcio que mejor se absorbe y se tolera es el aportado por la dieta por lo que la consejería nutricional destinada a facilitar a la persona el logro de sus propias metas en el consumo de preparados lácteos de bajo contenido en grasa, adquiere relevancia. La administración de preparados de calcio, se reserva para los casos en que, tras haber instruido a la mujer, no se consiga cubrir los requerimientos diarios.

Todas las sales de calcio tienen una eficacia aceptable y parece que se absorben y toleran mejor cuando se administran junto con lactosa, disminuyendo su absorción si se administran con exceso de grasa o fosfato. Por otra parte, no existen evidencias de que un aumento en la ingesta de calcio favorezca la litiasis renal $(10,18)$.

Otro aspecto de importancia nutricional de la mujer climatérica, corresponde a las necesidades de calcio las que se establecen en $1000 \mathrm{mg}$ al día durante la edad adulta hasta la menopausia. En ese momento el efecto de la deprivación hormonal conduce a un aumento de la pérdida de masa ósea. Además la capacidad de absorción del calcio en el intestino está disminuida, con lo que a partir de la menopausia se debe garantizar un aporte de calcio de $1500 \mathrm{mg}$ al día. Este aporte puede ser de 1000 $\mathrm{mg}$ al día en mujeres con terapia de reemplazo hormonal $(10,11,18)$.

La fibra resultó con una adecuación significativamente menor, reflejándose como factor de riesgo alimentario. Se explica debido a que el consumo de verduras fue significativamente bajo en ambas estaciones y el de frutas significativamente bajo en primavera verano. La educación alimentaria y la respectiva consejería acerca de la importancia del consumo de alimentos ricos en fibra constituirían una medida apropiada.

La encuesta utilizada consideró el agregado de sal a las comidas por lo que la variable sodio dentro del IAS consideró las dos fuentes: intrínseco y extrínseco, resultando en valores significativamente superiores a lo esperado pero con un puntaje del IAS superior a 7 considerado aceptable. Aunque en algunas mujeres el consumo fue alto, el valor promedio se explica tal vez por la conciencia de la mayoría de estas mujeres respecto del consumo de sal como factor de riesgo de hipertensión arterial. La OMS recomienda un consumo de sal no superior a 5 gramos al día aunque los consumos teóricos sugeridos corresponden a 2,4 gramos/día $(10,25,26)$.

La evaluación cualitativa de la dieta, muestra que dos tercios de las mujeres estudiadas necesitan realizar modificaciones en su alimentación. Los valores de adecuación de la dieta, indicador cuantitativo de utilidad para medir el grado de suficiencia de la dieta, aparecen mayoritariamente adecuados o definitivamente insuficientes con excepción de las proteínas y el hierro. En la consulta nutricional normalmente se trabaja con este último indicador, el cual se estaría manifestando poco apropiado como contribuyente del diagnóstico nutri- 
cional. De este modo el IAS adquiere importancia en estudios nutricionales más acabados. Sin embargo, por su complejidad, expertos aconsejan no aplicar el IAS a nivel individual $(12,24)$.

En Estados Unidos en grupos de población, se ha descrito el valor predictivo del IAS para ciertas enfermedades crónico degenerativas asociado a biomarcadores plasmáticos (27-29). El aquí aplicado corresponde a una adaptación de acuerdo a las recomendaciones del Ministerio de Salud de Chile para la ingesta de porciones de alimentos para la mujer adulta (11). Su aplicación resultó muy adecuada debido al diseño del estudio el que consideró registros dietarios de 14 días a cada mujer lo que permite obtener tendencias en un periodo de tiempo más prolongado en las ingestas. Los puntajes obtenidos por cada una de las 10 variables, estuvieron bajo el 65 $\%$ de lo esperado tanto en primavera-verano, como en otoño-invierno, mostrando la necesidad de intervención alimentaria y nutricional.

Se concluye que las ingestas energéticas de estas mujeres son bajas o adecuadas, y la calidad de la dieta medida por el IAS en los días estadísticamente representativos, refleja que requiere de cambios especialmente en cuanto a aumentar el consumo de frutas, lácteos, mejorar la variedad de alimentos y disminuir la cantidad de azúcar y sal, indicando la necesidad de intervención profesional.

\section{RESUMEN}

Para describir la calidad de la dieta, se analizó la adecuación de energía y nutrientes y el Indice de Alimentación Saludable (IAS), en 23 mujeres climatéricas de un universo de 268, de edades entre 47 y 61 años, pertenecientes al estamento administrativo de la Universidad de Concepción. Para la evaluación dietética, se utilizó la técnica de encuesta de registro diario de consumo de alimentos por 7 días consecutivos, separando los registros en dos períodos del año: Primavera-Verano y Otoño-Invierno. Se observó una adecuación normal de calorías en 12 de las 23 mujeres y adecuaciones significativamente menores en hidratos de carbono, ácidos grasos monosaturados, colesterol, calcio y fibra. Significativamente mayores en proteínas, vitamina $\mathrm{C}$ y hierro. El puntaje promedio del IAS en ambos períodos fue de $61,8 \pm 12,5(\mathrm{p}>0.05)$ clasificando la dieta en la categoría "necesidad de cambio" y un cumplimiento de las metas alimentarias inferior al $65 \%$. Los puntajes más bajos correspondieron a azúcar, lácteos y frutas. Se concluye que la alimentación de estas mujeres requiere de intervención alimentaria para mejorar la adecuación de nutrientes y el Indice de Alimentación Saludable.

Palabras claves: climaterio, encuesta ingesta de alimentos, nutrientes.

Dirigir la correspondencia a:

Profesora

Eliana Durán Fernández.

Departamento de Bromatología,

Nutrición y Dietética

Facultad de Farmacia,

Universidad de Concepción.

Casilla 237

Concepción

Fono: 41-204544 Fax: 41-210568

E-mail: eduran@udec.cl

Agradecimientos: Proyecto de Investigación PI $\mathrm{N}^{\circ}$ 202.073.024-1. Financiado por la Dirección de Investigación de la Universidad de Concepción.

\section{BIBLIOGRAFÍA}

1. World Health Organization (WHO). Report Meeting of the Subcomittees of the WPACHR on Enviromental Health, Health Promotion, on Health Sistems Research. Manilla, Philippines; 1996. Regional office for the Western Pacific. Report Series n RS/95/ GE/29 (PHL).

2. Porcile A, Aedo S, Florin M. Impacto de una guía para la atención del climaterio en la red de atención integral del Servicio de Salud Metropolitano Oriente de Chile. IV Congreso Latinoamericano de Climaterio y Menopausia FLASCYM 2004:25.

3. Aedo M, Sócrates, Porcile J, Arnaldo y Irribarra A, Cristina. Calidad de vida relacionada con el climaterio en una población chilena de mujeres saludables. Rev Chil Obstet Ginecol 2006;71(6):402-409.

4. Schneider HPG. The quality of life in the postmenopausal woman. Best Pract Res Clin Obstet Gynaecol 2002;16(3):395-409

5. INE. Chile: Estimaciones y Proyecciones de Población por Sexo y Edad. País Urbano y Rural, 1990-2010 en www. Ine.cl/canales/chile_estadístico/demografía.

6. Pisabarro R. Metabolismo y climaterio: la visión de un endocrinólogo. Rev Med Uruguay 2000; 16:144151.

7. Gómez G, Alfaro S. et al Nutrición y menopausia. Rev Colombiana Menopausia. 2002; 8 (2).

8. FAO/OMS/UNU, Necesidades de energía y proteínas. Informe de una reunión de expertos FAO/OMS/ UNU. OMS, Ginebra, 1985.

9. INE, Estadísticas demográficas y vitales, Chile, 1995-2020 en www.ine.cl.

10. Candelas R, Altarriba ML, López A. Nutrición en el 
climaterio. Informe de SVMFYC, Valencia, España. En www.svmfyc.org

11. Burrows R, Castillo C, Atalah E, Uauy R. Guias de alimentación para la mujer. Santiago, Chile 2001.

12. Pinheiro A, Atalah E. Propuesta de una metodología de análisis de la calidad global de la alimentación. Rev Méd Chile 2005; 133 (2): 175-182.

13. Urteaga $C$, Pinheiro A. Investigación alimentaria: consideraciones prácticas para mejorar la confiabilidad de los datos. Rev Chil Nutr 2003; 30: 235-42

14. Durán E, Soto D, Labraña AM, Pradenas F. Estacionalidad y días de la semana como factores de riesgo de la dieta de mujeres adultas. Rev Chil Nutr 2005; 32 (3): 254-261.

15. Durnin, J and Womersley J. Br J Nutr 1974;32:7797

16. Nacional Research Council (NRC). Subcomité on the tenth edition of the RDAs. Recommended dietary alowances 10th ed. Washington: National Academy Press; 1989.

17. Asenjo G, Durán E. Desarrollo de un software para evaluación y adecuación de la dieta. Versión actualizada. Universidad de Concepción, 2003

18. NIH Consensus conference. Optimal calcium intake. NIH Consensus Developmental Panel on Optimal calcium Intake. JAMA 1994;272:1942-1948

19. Dietary Referente Intakes for Macro and Micronutrients 1997-2001 in www.nap.edu.

20. Neves R, Nunes M, Mendes J. Avaliação do estado nutricional e do consumo alimentar de mulheres no climatério. Rev Assoc Med Bras 2003; 49 (1):
91-5

21. Wing R, Matthews K, Kuller L, Mailahn E, Platinga P. Weight gain at the time of menopause. Arch Intern Med 1991; 151: 97-103

22. Poehlman E, Michael J, Gardner A. Changes en energy balance and body composition at menopause: a controlled longitudinal study. Ann Intern Med 1995; 123: 673-5

23. Williamson D. The 10-year incidente of overweigth gain in U.S. adults. Arch Intern Med 1990; 150: 655-72.

24. MINSAL-Chile. Manual de Orientación en Atención Integral con Enfoque Familiar. Departamento Ciclo Vital, 2007.

25. Quiles J. Consejos y cuidados nutricionales en el climaterio. Alimentación, Nutrición y Salud 2000; 7 (2): 37-50.

26. Durán E, Soto D, Asenjo G, Labraña AM, Quiróz V, Pradenas F. Ingesta dietaria de sodio, potasio y calcio en embarazadas normotensas. Rev Chil Nutr 2002; 29:40-6).

27. Nicklas T. Assessing diet quality in children and adolescents. J Am Diet 2004;104: 1383-4.

28. Mc Cullough M, Feskanich D, Rimm E et al. Adherence to the Dietary Guidelines for Americans and risk of major chronic disease in men. Am J Clin Nutr 2000; 72: 1223-31.

29. Mc Cullough M, Feskanioch D, Stampfer M et al. Adherence to the Dietary Guidelines for Americans and risk of major chronic disease in women. Am J Clin Nutr 2000; 72: 1214-22. 